\title{
A case of leukocytoclastic vasculitis associated with anti-tumor necrosis factor therapy
}

\author{
Dorota Sikorska ${ }^{1}$, Renata Marcinkowska-Pięta ${ }^{1}$, Ewa Mojs ${ }^{1}$, Ryszard Żaba² ${ }^{2}$ Zygmunt Adamski², \\ Włodzimierz Samborski ${ }^{1}$
}

'Department of Rheumatology and Rehabilitation, Poznan University of Medical Sciences, Poznan, Poland ${ }^{2}$ Department of Dermatology, Poznan University of Medical Sciences, Poznan, Poland

Adv Dermatol Allergol 2018; XXXV (3): 323-324

DOI: https://doi.org/10.5114/ada.2018.76230

Tumor necrosis factor- $\alpha$ (TNF- $\alpha$ ) is one of the known cytokines, which is implicated in the pathogenesis of many chronic inflammatory diseases, including rheumatoid arthritis and ankylosing spondylitis [1]. Tumor necrosis factor- $\alpha$ inhibitors are increasingly used for the treatment of rheumatoid diseases. Anti-TNF- $\alpha$ therapy is generally well tolerated. However, there are a growing number of reports of the development of adverse events related to anti-TNF- $\alpha$ agents [2, 3]. A number of cutaneous side effects have been reported with anti-TNF- $\alpha$ therapy, including psoriatic eruption, lupus-like disorders and vasculitis [4]. However, only a few cases of leukocytoclastic vasculitis have been reported and they are rarely related to adalimumab [5-7].

Here we report a case of a patient with ankylosing spondylitis, who developed leukocytoclastic vasculitis after adalimumab treatment.

A 23-year-old male patient had a 13-year history of arthralgia, especially the knee joints and spine. In addition, he underwent surgical treatment of bilateral cleft lip and palate and surgical treatment of pyloric stenosis. In 2010, juvenile idiopathic arthritis was diagnosed. The patient received therapy with sulfasalazine (in 2010-2013), methotrexate (in 2010-2011) and periodically glucocorticoids and non-steroidal anti-inflammatory drugs, with no satisfactory treatment effects. In 2013, the patient was hospitalized in the Department of Rheumatology. The laboratory tests showed elevated inflammatory markers, with negative rheumatoid factor and positive HLA-B27 antigen. Radiological studies showed typical inflammation in the sacroiliac joints. The ankylosing spondylitis was diagnosed. The patient received adalimumab of $40 \mathrm{mg}$ subcutaneously every 2 weeks and showed good response to therapy. After 35 months of treatment, the patient complained of pain and swollen ankles and appearance of skin lesions. Blotchy rash initially included the ankle and then spread to the entire lower limbs. Dermatological examination revealed purpura with erosions and blisters filled with the contents of seroblood (Figures 1, 2). There were no other systemic signs, however the patient was undergoing dental treatment. The laboratory tests showed no inflammatory markers, and results of all basic laboratory tests (complete blood count, renal, liver and thyroid function, urinalysis) were within the reference values. However, the laboratory tests showed an anti-nuclear antibody (ANA) 1/320 of a granular type of lighting. The anti-dsDNA, anti-extractable nuclear antigen (ENA) antibodies and anti-neutrophil cytoplasmic antibodies (ANCA: pANCA and cANCA) were negative. Moreover, HIV, HCV and HBV infections were excluded. Histopathological examination of the skin was performed. Based on the clinical and histopathological findings, the patient was diagnosed with leukocytoclastic vasculitis, probably due to therapy with adalimumab. Adalimumab was discontinued and methylprednisolone ( $8 \mathrm{mg} /$ day) and cefuroxime $(500 \mathrm{mg} /$ day because of the patient's dental treatment) were prescribed. Also local treatment was prescribed (betamethasone and gentamycin). After 4 weeks the patient had complete resolution of symptoms.

The available safety data on autoimmune diseases induced by TNF- $\alpha$ inhibitors rely mainly on case reports, and information regarding their management and clinical significance is very limited. Ramos-Casals et al. have described the clinical characteristics of 113 patients who developed vasculitis (the most frequent type of vasculitis was leukocytoclastic vasculitis - in 79 cases) after receiving anti-TNF agents (etanercept in 59 cases, infliximab in 47, adalimumab in 5, and other agents in 2). However, the association of leukocytoclastic vasculitis and different anti-TNF inhibitors (human vs. humanized vs. chimeric) is unclear [8].

Address for correspondence: Dorota Sikorska MD, PhD, Department of Rheumatology and Rehabilitation,

Poznan University of Medical Sciences, 28 Czerwca 1956, 60-375 Poznan, Poland, phone: +48 693 484 352, e-mail: dorota_s8@wp.p Received: 7.12.2016, accepted: 27.03.2017. 


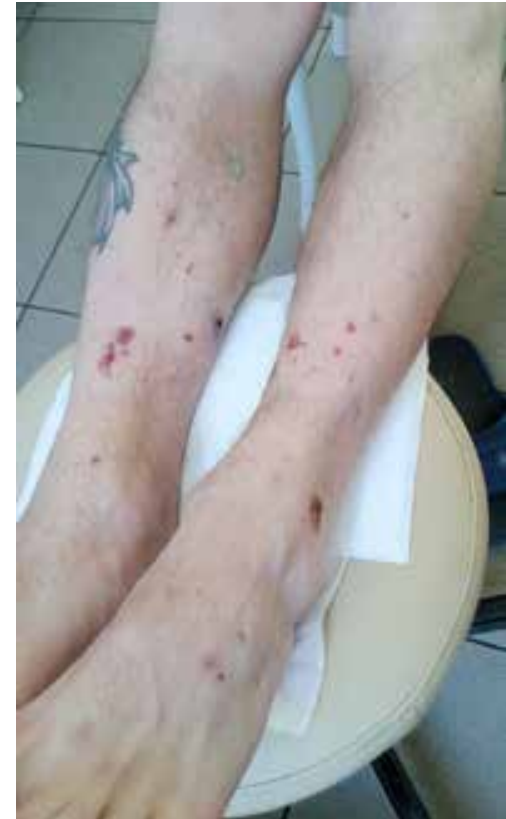

Figure 1. Leukocytoclastic vasculitis associated with antiTNF- $\alpha$ inhibitor

The pathogenic mechanism for development of druginduced leukocytoclastic vasculitis is not fully defined $[9,10]$. Probably the auto-antibody against anti-TNF- $\alpha$ inhibitors (like adalimumab) may be related to the pathogenesis of this side effect $[11,12]$. It has been suggested that immune complexes, such TNF- $\alpha$ /TNF- $\alpha$-antibody are deposited in the small capillary, and can activate type III hypersensitivity reaction [13]. It has been also reported that the appearance of anti-drug antibody is closely related to the occurrence of ANA and paradoxical inflammations [13]. Usually after discontinuation of the TNF- $\alpha$ inhibitor, patients have complete resolution of symptoms [12].

In conclusion, drug-induced leukocytoclastic vasculitis is a rare complication, however early diagnosis is critical to successful patient outcome.

\section{Conflict of interest}

The authors declare no conflict of interest.

\section{References}

1. Chew AL, Bennett A, Smith CH, et al. Successful treatment of severe psoriasis and psoriatic arthritis with adalimumab. Br J Dermatol 2004; 151: 492-6.

2. Fiorino G, Danese S, Pariente B, Allez M. Paradoxical immune-mediated inflammation in inflammatory bowel disease patients receiving anti-TNF-alpha agents. Autoimmun Rev 2014; 13: 15-9.

3. Perez-Alvarez R, Perez-de-Lis M, Ramos-Casals M. Biologicsinduced autoimmune diseases. Curr Opin Rheumatol 2013; 25: 56-64.

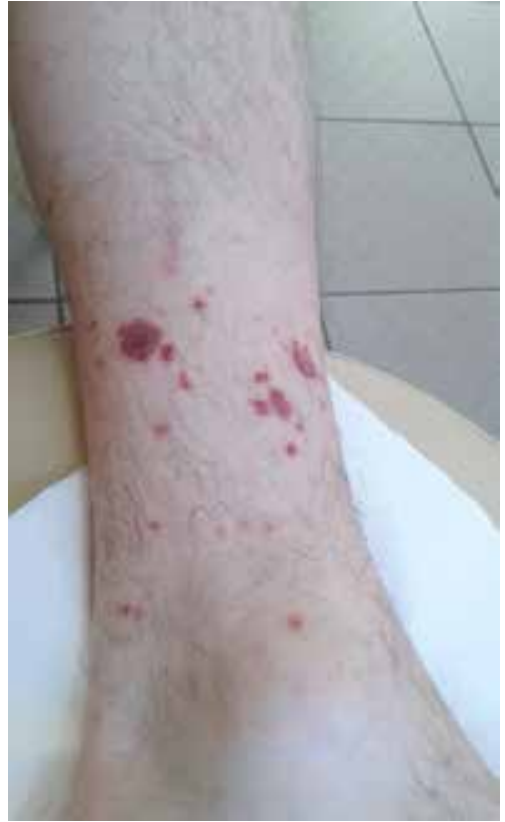

Figure 2. Leukocytoclastic vasculitis associated with antiTNF- $\alpha$ inhibitor

4. Exarchou SA, Voulgari PV, Markatseli TE, et al. Immunemediated skin lesions in patients treated with anti-tumour necrosis factor alpha inhibitors. Scand J Rheumatol 2009; 38: 328-31.

5. Amarante CF, Acedo LM, Rabay FM, et al. Drug-induced lupus with leukocytoclastic vasculitis: a rare expression associated with adalimumab. An Bras Dermatol 2015; 90 (3 Suppl. 1): 121-4.

6. Ramos-Casals M, Brito-Zeron P, Munoz S, et al. Autoimmune diseases induced by TNF-targeted therapies: analysis of 233 cases. Medicine 2007; 86: 242-51.

7. Mohan N, Edwards ET, Cupps TR, et al. Leukocytoclastic vasculitis associated with tumor necrosis factor-alpha blocking agents. J Rheumatol 2004; 31: 1955-8.

8. Ramos-Casals M, Brito-Zeron P, Soto MJ, et al. Autoimmune diseases induced by TNF-targeted therapies. Best Pract Res Clin Rheumatol 2008; 22: 847-61.

9. Cakiter AU, Kucuk OS, Ozkaya DB, et al. Demographic characteristics, aetiology, and assessment of treatment options in leukocytoclastic vasculitis. Adv Dermatol Allergol 2017; 34: 104-9.

10. Gambichler T, Kulik MA, Skrygan M, et al. Cutaneous leukocytoclastic vasculitis: the role of lymphocytes and related immune markers. Adv Dermatol Allergol 2017; 34: 299-305.

11. Baert F, Noman M, Vermeire S, et al. Influence of immunogenicity on the long-term efficacy of infliximab in Crohn's disease. N Engl J Med 2003; 348: 601-8.

12. Numakura T, Tamada T, Nara M, et al. Simultaneous development of sarcoidosis and cutaneous vasculitis in a patient with refractory Crohn's disease during infliximab therapy. BMC Pulmon Med 2016; 16: 30.

13. Pink AE, Fonia A, Allen $\mathrm{MH}$, et al. Antinuclear antibodies associate with loss of response to antitumour necrosis factoralpha therapy in psoriasis: a retrospective, observational study. Br J Dermatol 2010; 162: 780-5. 